Revista de Matemática: TeOría y APliCACiones 2017 24(2) : 227-238

CIMPA - UCR ISSN: 1409-2433 (PRINT), 2215-3373 (ONLINE)

\title{
SOBRE EL TEOREMA DE FUBINI PARA CONJUNTOS DE MEDIDA CERO EN MEDIDAS VECTORIALES
}

\section{ON FUBINI'S THEOREM FOR NULL SETS IN VECTOR MEASURES}

\author{
ARMAndo Montilla*
}

Received: 22/Jun/2016; Revised: 17/Apr/2017;

Accepted: 28/Apr/2017

Revista de Matemática: Teoría y Aplicaciones is licensed under a Creative Commons
Reconocimiento-NoComercial-Compartirigual 4.0 International License.
Creado a partir de la obra en http://www.revistas.ucr.ac.cr/index.php/matematica

*Universidad de Los Andes, Núcleo Universitario "Rafael Rangel”, Trujillo, Venezuela. EMail: armandom@ula.ve 


\title{
Resumen
}

En este artículo presentamos una versión del Teorema de Fubini para conjuntos de medida cero en el contexto de medidas vectoriales, en el estilo de la demostración clásica para conjuntos del plano euclidiano; a saber: Sean $X$ y $Y$ espacios topológicos de Hausdorff localmente compactos $\mathrm{y}, \mu \mathrm{y} \nu$ medidas vectoriales regulares de variación acotada en las $\sigma$-álgebras de Baire $\mathcal{B}_{0}(X)$ y $\mathcal{B}_{0}(Y)$, respectivamente. Si $A \subset X \otimes Y$, entonces $|\mu \otimes \nu|(A)=0$ si sólo si

$$
|\mu|\left\{x \in X: A_{x} \text { no es de medida cero }\right\}=0,
$$

donde $A_{x}=\{y \in Y:(x, y) \in A\}$.

Palabras clave: medida vectorial; medida cero; medida producto; teorema de Fubini.

\begin{abstract}
In this paper we prove a version of Fubini's Theorem for null sets in the context of vector measures, in the spirit of the classical proof for sets in the Euclidean plane, namely: Let $X$ and $Y$ be locally compact Hausdorff topological spaces and let $\mu$ and $\nu$ be regular vector measures on the Baire $\sigma$-algebras $\mathcal{B}_{0}(X)$ and $\mathcal{B}_{0}(Y)$, respectively. If $A \subset X \times Y$, then $|\mu \otimes \nu|(A)=0$ if and only if

$$
|\mu|\left(\left\{x \in X: A_{x} \text { is not a null set }\right\}\right)=0,
$$

where $A_{x}=\{y \in Y:(x, y) \in A\}$.
\end{abstract}

Keywords: vector measure; zero measure; product measure; Fubini's theorem.

Mathematics Subject Classification: 26B15, 28A25.

\section{Reseña histórica}

En 1904 Lebesgue [3] extiende la teoría de integración de Riemman. En la teoría de la medida según Lebesgue se define la medida de un subconjunto de $\mathbb{R}$ en términos de cubiertas del conjunto por sucesiones de intervalos y para un subconjunto de $\mathbb{R}^{2}$ en términos de cubiertas por sucesiones de rectángulos. Surge de manera natural una interrogante : ¿qué relación existe entre una y otra medida? Aunque Lebesgue no resolvió completamente esta interrogante, su teoría sentó las bases para que muchos otros matemáticos trabajaran en este aspecto y uno de ellos fué Fubini y en 1907 publica sus resultados en este sendido sobre integrales iteradas [4]. Un aspecto particular del teorema de Fubini sería: si A tiene medida 
bidimensional tendrán cada sección de A perpendicular a los ejes una medida unidimensional, Fubini afirma que si, excepto en un conjunto unidimensional de medida cero y en el caso particular de que A tenga medida bidimensional cero cada sección tiene medida unidimensional cero excepto en un conjnunto de medida cero. Se ha demostrado que $A$ tiene medida cero si sólo si cada sección tiene medida cero casi siempre. Ahora en el presente artículo se presenta una versión de esta prueba en contextos mas generales; cuando el espacio de medida son los borelianos de un espacio topológico de Hausdorff localmente compacto y las medidas, medidas vectoriales regulares de Borel y se usa un resultado que aparece en un articulo publicado por S.K Berberian en 1966 [1].

\section{Introducción}

En Análisis clásico el nombre de Fubini suele asociarse a un resultado acerca de la relación que existe entre la integral sobre un producto de espacios y la integral definida sobre los factores de tal producto, ver por ejemplo [10] y [11]. Pero existe otro resultado sutil, conocido con el nombre de Fubini, que explora la relación entre la medida de conjuntos del espacio producto y algunos subconjuntos de los factores. Así, por ejemplo, en [3] se presenta dicho resultado en el contexto de la medida de Lebesgue para el plano $\mathbb{R}^{2}$; tal resultado establece una condición necesaria y suficiente para que un conjunto $A \subset \mathbb{R}^{2}$ tenga medida cero dependiendo de la $x$-sección $A_{x}=\{y \in \mathbb{R}:(x, y) \in A\}$; formalmente:

Teorema de Fubini en el plano. Para un conjunto medible $A \subset \mathbb{R}^{2}$, las siguientes proposiciones son equivalentes:

(a) A es un conjunto de medida cero en $\mathbb{R}^{2}$;

(b) $A_{x}$ es un conjunto de medida cero en $\mathbb{R}$ para casi todo $x \in \mathbb{R}$.

La demostración de la implicación (a) $\Rightarrow$ (b) de este teorema se basa en la prueba dada en [9], mientras que la otra es trabajada con cierto detalle en el artículo. Este teorema puede ser fácilmente extendido a espacios euclídeos en general e inclusive, como se indica en [3], puede ser planteado en un escenario más general con ciertas hipótesis: "que los espacios topológicos involucrados como factores $X$ e $Y$ sean de Hausdorff localmente compactos y que las medidas vectoriales asociadas sean regulares sobre las respectivas $\sigma$-álgebras de Baire $\mathcal{B}_{0}(X)$ y $\mathcal{B}_{0}(Y)$ ". Por supuesto, en este esquema si $A \subset X \times Y$, la $x$-sección $A_{x}$ es el conjunto $\{y \in Y:(x, y) \in A\}$. 
Teorema de Fubini. Sean X y $Y$ espacios topológicos de Hausdorff localmente compactos, $\mu$ y $\nu$ medidas regulares vectoriales de variación acotada en las $\sigma$ álgebras de Baire de $\mathcal{B}_{0}(X)$ y $\mathcal{B}_{0}(Y)$, respectivamente. Entonces las siguientes proposiciones son equivalentes:

(a) A es un conjunto de medida cero en $\mathcal{B}_{0}(X) \times \mathcal{B}_{0}(Y)$;

(b) $A_{x}$ es un conjunto de medida cero en $\mathcal{B}_{0}(Y)$ para casi todo $x \in X$.

En el presente artículo se expone una demostración de esta versión del Teorema de Fubini, en el espíritu de [3] y [9]. En nuestra presentación, la implicación (a) $\Rightarrow$ (b) es la Proposición 8 mientras que la proposición (b) $\Rightarrow$ (a) es la Proposición 9. Las hipótesis de naturaleza topológica permiten aplicar una variante del "lema del tubo" (Teorema 2) y las de regularidad de las medida garantizan la acotación de las medidas de conjuntos compactos, lo cual permite seguir el esquema de la prueba en espacios euclídeos.

\section{Preliminares}

Esta sección contiene algunos resultados clásicos que son importantes en el desarrollo de la prueba del Teorema de Fubini. Para empezar, tenemos dos teoremas de topología.

Teorema 1 Sea $(X, \mathcal{T})$ un espacio de Hausdorff. Si $\left\{N_{\alpha}\right\}_{\alpha \in I}$ es la colección de todos los entornos de un punto $x \in X$, entonces

$$
\bigcap_{\alpha \in I} N_{\alpha}=\{x\}
$$

Demostración. Supongamos que $\bigcap_{\alpha \in I} N_{\alpha} \neq\{x\}$, entonces existe $y \in$ $\bigcap_{\alpha \in I} N_{\alpha}$ tal que $y \neq x$. Como el espacio es de Hausdorff existen vecindades $U_{x}$ de $x$ y $U_{y}$ de $y$, tales que $U_{x} \cap U_{y}=\emptyset$, de manera que $U_{x}$ es una vecindad de $x$ que no contiene a $y$ y esto contradice el hecho de que $y$ pertenece a toda vecindad de $x$.

En lo sucesivo si $(X, \mathcal{T})$ y $(Y, \mathcal{S})$ son dos espacios topológicos, $X \times Y$ es el espacio producto, y la $x$-sección de $A \subset X \times Y$, denotada por $A_{x}$ se define como:

$$
A_{x}=\{y \in Y:(x, y) \in A\}
$$


Teorema 2 Sean $(X, \mathcal{T})$ y $(Y, \mathcal{S})$ dos espacios topológicos, siendo $(X, \mathcal{T})$ un espacio de Hausdorff. Sea $A \subset X \times Y$ un conjunto compacto. Supongamos que $x \in X$ y que existe $S \in \mathcal{S}$ tal que $A_{x} \subset S$, entonces existe un $T \in \mathcal{T}$, tal que $A_{y} \subset S$ para todo $y \in T$, es decir $A \cap(T \times Y) \subseteq T \times S$.

Demostración. Sea $J$ el conjunto dirigido de los entornos de $x$ y supongamos que la tesis del teorema es falsa, es decir que para todo entorno de $x, N_{x}$, existe $x_{i} \in N_{x}$ tal que $A_{x_{i}}$ no está contenida en $S$, es decir, existe $y_{i} \in Y \operatorname{con}\left(x_{i}, y_{i}\right) \in$ $A$, y $y_{i} \notin S$. Como $X$ es de Hausdorff, la red $\left(x_{i}\right)_{i \in J}$ converge a un único punto $x,\left(x_{i}\right)_{i \in J} \rightarrow x$, y como $A$ es compacto la red $\left(x_{i}, y_{i}\right)_{i \in J}$ tiene una subred convergente, digamos $\left(x_{i}, y_{i}\right)_{i \in K}$ y $K$ cofinal en $J$, por lo que

$$
\left(x_{i}, y_{i}\right)_{i \in K} \rightarrow(x, y) \text {. }
$$

De manera que $y \in A_{x}$ y $y \notin S$, ya que $y_{i} \notin S$ para todo $i \in K$, esta contradicción prueba que el teorema es cierto.

A continuación consideramos algunos elementos de teoría de la medida involucrados con las hipótesis del Teorema de Fubini. Empezamos con tres definiciones.

Definición 1 Sea $X$ un espacio topológico Hausdorff localmente compacto. Se define la $\sigma$-álgebra de Borel en $X$, denotada como $\mathcal{B}(X)$, como la $\sigma$-álgebra generada por los compactos, es decir la intersección de todas las $\sigma$-álgebras que contienen los compactos. Si $\mu$ es una medida vectorial $\sigma$-aditiva, definida en $\mathcal{B}(X)$ con valores en un espacio normado $Z$ se dice que es regular si para todo $\epsilon>0$ y $E \in \mathcal{B}$ existen un compacto $K$ y un abierto $U$, tal que $K \subset E \subset U$ $y|\mu(F)|<\epsilon$ para todo $F \in \mathcal{B}$ con $F \subset U \backslash K$.

Definición 2 Sea $X$ un espacio topológico de Hausdorff localmente compacto. Se define la $\sigma$-álgebra de Baire en $X$, denotada como $\mathcal{B}_{0}(X)$, como la $\sigma$-álgebra generada por los compactos $G_{\delta}$ es decir la intersección de todas las $\sigma$-álgebras que contienen los compactos $G_{\delta}$. Si $\mu$ es una medida vectorial $\sigma$-aditiva, definida en $\mathcal{B}_{0}(X)$ con valores en un espacio normado $Z$, se dice que es regular si para todo $\epsilon>0$ y $E \in \mathcal{B}_{0}$ existen un compacto $K, G_{\delta}$ y un abierto $U$, tal que $K \subset E \subset U y|\mu(F)|<\epsilon$ para todo $F \in \mathcal{B}_{0}$ con $F \subset U \backslash K$.

En relación con la definición anterior hay una redundancia, aunque no muy clara y es por el hecho de que toda medida vectorial de Baire con valores en un espacio normado es regular.

En lo sucesivo $\mathcal{B}$ denota cualquiera de las $\sigma$-álgebras $\mathcal{B}(X)$ o $\mathcal{B}_{0}(X), \mu$ es una medida vectorial $\sigma$-aditiva en $\mathcal{B}$, la variación de $\mu$ se denota $|\mu|$, donde $|\mu|(A)=\sup \sum_{i=1}^{n}\left|\mu\left(B_{i}\right)\right|$ donde $\left\{B_{i}\right\}_{i=1}^{n}$ es una partición dijunta de $A$. 
Es de hacer notar que en el caso de que $X$ sea metrizable, $\mathcal{B}(X)=\mathcal{B}_{0}(X)$.

La siguiente propiedad, que establece una relación entre la regularidad de la medida vectorial y la regularidad de su variación que en este caso es una medida escalar acotada.

Teorema $3 \mu: \mathcal{B} \rightarrow Z$ es regular si y sólo si $|\mu|: \mathcal{B} \rightarrow \mathbb{R}$ es una medida regular.

Demostración. Si $|\mu|$ es regular de [2], p.314, $\mu$ es regular. Reciprocamente si $\mu$ es regular entonces $|\mu|$ es una medida escalar definida en $\mathcal{B}$, por lo que es regular.

En el siguiente resultado, es una consecuencia del teorema anterior.

Teorema 4 Si $X$ es un espacio topológico localmente compacto y $\mu$ es una medida vectorial en $\mathcal{B}$, regular con valores en un espacio normado $Z$ y de variación acotada, entonces para cada compacto $K$ en $\mathcal{B}$, existe un abierto $U$ en $X$ tal que $K \subset U y|\mu|(U)<\infty$.

Como en el Teorema 4 , en las siguientes definiciones $\mathcal{B}$ denota cualquiera de las $\sigma$-álgebras $\mathcal{B}(X), \mathcal{B}_{0}(X)$ o $\mathcal{B}_{w}(X)$, en forma respectiva.

Definición 3 Sean $X$, un espacio topológico, $Y$ un espacio normado $\mu$ una medida vectorial, $\mu: \mathcal{B} \rightarrow Y$ y $A \subset X$. Se dice que la colección $\left\{K_{i}\right\}_{i=1}^{\infty}$ con $K_{i} \in \mathcal{B}$ es un cubrimiento de A si para cada $x \in A$ existe al menos un $K_{i}$ tal que $x \in K_{i}$, es decir $A \subset \bigcup_{i=1}^{\infty}\left\{K_{i}\right\}$, y decimos que la colección $\left\{K_{i}\right\}_{i=1}^{\infty}$ con $K_{i} \in \mathcal{B}(X)$ es un cubrimiento infinitamente infinito de $A$ si para cada $x \in A$ existe una subcolección $\left\{K_{i_{j}}\right\}_{j=1}^{\infty}$, tal que $x \in K_{i_{j}}$ para todo $j \in \mathbb{N}$.

Finalmente tenemos la extensión del concepto de "medida cero" para el caso de medidas vectoriales.

Sea $X$ un espacio topológico, $Y$ un espacio normado y $\mu$ una medida regular vectorial, $\mu: \mathcal{B} \rightarrow Y$. Se dice que $A \subset X$ es de $\mu$-medida cero si para todo $\epsilon>0$ existe un cubrimiento $\left\{U_{i}\right\}_{i=1}^{\infty}$ de conjuntos abiertos, tal que

$$
\sum_{i=1}^{\infty}|\mu|\left(U_{i}\right)<\epsilon .
$$

En este caso escribimos $\mu(A)=0$ sin que ello signifique necesariamente que $A$ es medible.

Con el mismo convenio de notación del Teorema 4 tenemos el siguiente resultado. 
Teorema 5 Sean $X$ un espacio topológico, $Y$ un espacio normado y $\mu$ una medida vectorial regular, $\mu: \mathcal{B} \rightarrow Y$. $E \subset X$, es de $\mu$-medida cero si y sólo si existe un cubrimiento infinitamente infinito de $E,\left\{U_{i}\right\}_{i=1}^{\infty}$, de conjuntos abiertos, tal que $\sum_{i=1}^{\infty}|\mu|\left(U_{i}\right)$ es convergente.

Demostración. Supongamos que $E$ es de medida cero, por lo tanto para cada $n \in \mathbb{N}$, existe un cubrimiento abierto $\left\{K_{m}(n)\right\}_{m=1}^{\infty}$ de $E$, tal que

$$
\sum_{m=1}^{\infty}|\mu|\left(K_{m}(n)\right)<\frac{1}{2^{n}}
$$

Evidentemente $\left\{K_{m}(n)\right\}_{m, n=1}^{\infty}$ es un cubrimiento abierto infinitamente infinito de $E$, que puede se dirigido por los naturales, es decir,

$$
\left\{K_{m}(n)\right\}_{n, m=1}^{\infty}=\left\{U_{i}\right\}_{i=1}^{\infty},
$$

donde $U_{i}=K_{m}(n)$ para algunos $m, n \in \mathbb{N}$. Sea

$$
C_{n}=\bigcup_{m=1}^{\infty} K_{m}(n)
$$

luego

$$
|\mu|\left(C_{n}\right)=|\mu|\left(\bigcup_{m=1}^{\infty} K_{m}(n)\right) \leq \sum_{m=1}^{\infty}|\mu|\left(K_{m}(n)\right)<1 / 2^{n}
$$

y $U_{i} \subset C_{n}$ para algún $n \in \mathbb{Z}$.

Ahora

$$
|\mu|\left(\bigcup_{i=1}^{\infty} U_{i}\right)=|\mu|\left(\bigcup_{i=1}^{\infty} C_{i}\right) \leq \sum_{i=1}^{\infty}|\mu|\left(C_{i}\right) \leq \sum_{i=1}^{\infty} 1 / 2^{n} \leq 1 .
$$

Para cada $i \in \mathbb{N}$ existe $n \in \mathbb{N}$ tal que $U_{i} \subset C_{n}$, por lo que $|\mu|\left(U_{i}\right) \leq|\mu|\left(C_{n}\right)$, y se tiene:

$$
\sum_{i=1}^{\infty}|\mu|\left(U_{i}\right) \leq \sum_{i=1}^{\infty}|\mu|\left(C_{n}\right) \leq 1
$$

Ahora supongamos que $\left\{U_{i}\right\}_{i=1}^{\infty}$ es un cubrimiento abierto infinitamente infinito de $E$, tal que $\sum_{i=1}^{\infty}|\mu|\left(U_{i}\right)<\infty$, entonces $\lim _{n \rightarrow \infty} \sum_{i=n}^{\infty}|\mu|\left(U_{i}\right)=0$, por 
lo tanto dado $\epsilon>0$ existe $n_{0} \in \mathbb{N}$ tal que $\sum_{k=n_{0}}^{\infty}|\mu|\left(U_{i}\right)<\epsilon$ y así se cumple que:

$$
|\mu|\left(\bigcup_{i=n_{0}}^{\infty} U_{i}\right) \leq \sum_{k=n_{0}}^{\infty}|\mu|\left(U_{i}\right)<\epsilon .
$$

Como el cubrimiento es infitamente infinito se cumple que

$$
E \subset \bigcup_{i=n_{0}}^{\infty} U_{i}
$$

Veamos un par de resultados que son fundamentales en la prueba del Teorema de Fubini. En primer lugar tenemos:

Teorema 6 Si $X$ y $Y$ son espacios topológicos de Hausdorff localmente compactos, entonces $\mathcal{B}_{0}(X) \times \mathcal{B}_{0}(Y)=\mathcal{B}_{0}(X \times Y)$.

Ver[5], p 222.

Si $\mu$ y $\nu$ son medidas vectoriales regulares en $\mathcal{B}_{0}(X)$ y $\mathcal{B}_{0}(Y)$ respectivamente, con valores en un algebra normada $Z$ se define $\mu \otimes \nu$ en

$$
\mathcal{C}=\left\{\left(U_{i}, V_{i}\right): U_{i} \in \mathcal{B}_{0}(X), V_{i} \in \mathcal{B}_{0}(Y)\right\},
$$

como $\mu \otimes \nu\left(U_{i}, V_{i}\right)=\mu\left(U_{i}\right) \cdot \nu\left(V_{i}\right)$, y esta se puede extender de manera única y regular a $\mathcal{B}_{0}(X) \times \mathcal{B}_{0}(Y)=\mathcal{B}_{0}(X \times Y)(\operatorname{ver}[6])$.

Del hecho de que $|U \cdot V| \leq|U| \cdot|V|$ al ser $Z$ un álgebra normada y de la regularidad se tiene el siguiente resultado:

Teorema 7 Si $\mu$ y son medidas vectoriales regulares en $\mathcal{B}_{0}(X)$ y $\mathcal{B}_{0}(Y)$ respectivamente, con valores en un algebra normada $Z$, entonces $E \subset \mathcal{B}_{0}(X) \times$ $\mathcal{B}_{0}(Y)$ es de medida $\mu \otimes \nu$ cero si y solo si para todo $\epsilon$ existe un cubrimiento $U_{i} \times V_{i}$ de E tal que $\sum_{i=1}^{\infty}|\mu|\left(U_{i}\right)|\nu|\left(V_{i}\right)<\epsilon$.

\section{El teorema de Fubini}

En esta sección presentamos la demostración del Teorema de Fubini. Notemos que decir que la sección $\nu\left(A_{x}\right)$ para casi todo $x$ es equivalente a decir que $\mu(E(A))=0$, donde el conjunto de excepción $E(A)$ está definido por

$$
E(A)=\left\{x \in X: A_{x} \text { no es un conjunto de medida cero }\right\} .
$$


Con esta terminología planteamos la implicación (a) $\Rightarrow$ (b) del Teorema de Fubini.

Proposición 8 Sean $X$ y $Y$ espacios topológicos de Hausdorff localmente compactos; $\mu$ y $\nu$ medidas regulares en las $\sigma$-álgebras de Baire $\mathcal{B}_{0}(X)$ y $\mathcal{B}_{0}(Y)$, respectivamente. Si $A \subset X \times Y$ con $\mu \otimes \nu(A)=0$, entonces $\mu(E(A))=0$.

Demostración. Sea $\varepsilon>0$, como $\mu \otimes \nu(A)=0$, por 7 se tiene que existe un cubrimiento infinito $\left\{U_{i} \times V_{i}\right\}_{i=1}^{\infty}$ de $A$ tal que

$$
\sum_{i=1}^{n}|\nu|\left(V_{i}\right)|\mu|\left(U_{i}\right)<\varepsilon
$$

Sea

$$
\varphi_{n}(x)=\sum_{i=1}^{n}|\nu|\left(V_{i}\right) \chi_{U_{i}}(x) .
$$

Claramente $\varphi_{i}-\varphi_{i-1}(x)=|\nu|\left(V_{i}\right) \chi_{U_{i}}(x)$. Por la propiedad telescópica

$$
\varphi_{n}=\sum_{i=1}^{n} \varphi_{i}-\varphi_{i-1}
$$

Aplicando el teorema de convergencia monótona se tiene que:

$$
\begin{aligned}
\int \varphi_{n} d|\mu| & =\int\left(\sum_{i=1}^{n} \varphi_{i}-\varphi_{i-1}\right) d|\mu| \\
& =\sum_{i=1}^{n} \int\left(\varphi_{i}-\varphi_{i-1}\right) d|\mu| \\
& =\sum_{i=1}^{n} \int|\nu|\left(V_{i}\right) \chi_{U_{i}} d|\mu| .
\end{aligned}
$$

De manera que

$$
\int \varphi_{n} d|\mu|=\sum_{i=1}^{n}|\nu|\left(V_{i}\right)|\mu|\left(U_{i}\right)<\epsilon .
$$

Ahora, sea $A_{i}=\left\{x: \varphi_{i}(x) \geq 1\right.$ y $\left.\varphi_{i-1}<1\right\}$. Luego $A_{i} \cap A_{j}=\emptyset$ para todo $i \neq j$ y $A_{i} \subset V_{i}$ para todo $i \in \mathbb{N}$. Cada $A_{i}$ es $\mu$-medible ya que $A_{i}=\varphi_{i}^{-1}\left([1, \infty) \cap \varphi_{i-1}^{-1}([0,1))\right.$.

Rev.Mate.Teor.Aplic. (ISSN print: 1409-2433; online: 2215-3373) Vol. 24(2): 227-238, July 2017 
Al ser $\varphi_{i}(x) \geq 1$ en $A_{i}$ para $n \geq i$, se tiene $\sum_{i=1}^{n}|\mu|\left(A_{i}\right) \leq \int \varphi_{n} d|\mu|$, para cada $n$, de manera que

$$
\sum_{i=1}^{\infty} \mu\left(A_{i}\right) \leq \epsilon
$$

La idea ahora es verificar que el conjunto $E=\left\{x: \nu\left(E_{x}\right) \neq 0\right\}$ es de $\mu$ medida cero, y para este fin se demuestra que este conjunto está contenido en la unión de $\operatorname{los} A_{i}$.

En efecto si $x \in E$ existe $y \in Y$ tal que $(x, y) \in A$, como $U_{i} \times V_{i}$ es una cubierta infinitamente infinita de $A$, existe una sucesión $i_{k}$, tal que $(x, y) \in$ $U_{i_{k}} \times V_{i_{k}}$, de manera que $x \in U_{i_{k}}$. Ahora si $y \in A_{x}, y \in V_{i_{k}}$ para infinitos valores de $k$, por lo que $V_{i_{k}}$ es una cubierta infinitamente infinita de $A_{x}$, de manera que la serie $\sum|\nu|\left(V_{i_{k}}\right)$ es divergente al ser $\nu\left(A_{x}\right) \neq 0$, de manera que $\lim _{n \rightarrow \infty} \varphi_{n}(x)=\infty$, y por lo tanto $x \in A_{i}$ para algún $i$. De lo anterior se concluye que $A_{x} \subset \cup A_{i}$.

Ahora procedemos a demostrar la implicación (b) $\Rightarrow$ (a) del Teorema de Fubini.

Proposición 9 Sean $X$ y $Y$ son espacios topológicos de Hausdorff localmente compactos y $\mu$ y $\nu$ medidas regulares en las $\sigma$-álgebras de Baire $\mathcal{B}_{0}(X)$ y $\mathcal{B}_{0}(Y)$, respectivamente. Si $A \subset X \times Y$ y $\mu(E(A))=0$, entonces $\mu \times \nu(A)=0$.

Demostración. Sin pérdida de generalidad podemos suponer que $\nu\left(A_{x}\right)=0$, es decir que el conjunto $E(A)=\emptyset$ y además que $A$ es compacto ya que la medida regular.

Dado $\delta>0$ arbitrario, demostraremos que $|\mu \times \nu|(A)<\delta$. Sea $L=\pi_{X}(A)$ la proyección de $A$ en $X$, como $\pi_{X}$ es continua se tiene que $L$ es un subconjunto compacto de $X$, por lo tanto existe un abierto $S$ en $X$ tal que $L \subset S$ y $|\mu|(S)<$ $\infty$. Elegimos $\epsilon>0$ de manera tal que $|\mu|(S) \epsilon<\delta$. Llamaremos a un conjunto $U \subset X \epsilon$-vecindad si existe un conjunto abierto $V \subset Y$ con $\nu(V)<\epsilon$ tal que $A \cap(U \times Y) \subset U \times V$.

Sea $\mathcal{G}$ la colección de las $\epsilon$-vecindades $G$ con $G \subset S$. Si $x \in L$, como $|\nu|\left(A_{x}\right)=0$, existe una vecindad $V \subset Y$ tal que $|\nu|(V)<\epsilon$ y $A_{x} \subset V$, por el Teorema 2 existe una vecindad $\epsilon$-buena $U$ en $X$, tal que

$$
A \cap(U \times Y) \subset U \times V .
$$

Es decir, $\mathcal{G}$ es un cubrimiento abierto de $L$, como éste es compacto se obtiene un subcubrimiento finito $\mathcal{G}^{\prime}=\left\{G_{1}, G_{2}, \ldots, G_{n}\right\}$; a partir de $\mathcal{G}^{\prime}$ podemos obtener 
un cubrimiento de $L$, el cual es finito y disjunto, considerando

$$
S_{k}=G_{k} \backslash \bigcup_{i<k} G_{i} \text { para } k=0, \ldots, n .
$$

Por supuesto, tenemos que

$$
\bigcup_{k=0}^{n} S_{k} \subset S
$$

Ahora, para cada $k$ podemos obtener un abierto $V_{k} \subset Y \operatorname{con} \nu\left(V_{k}\right)<\epsilon$ y tal que:

$$
A \cap\left(S_{k} \times Y\right) \subset S_{k} \times V_{k} .
$$

Con esta elección de $\epsilon$ se tiene que

$$
\begin{aligned}
|\mu \times \nu|(A)= & |\mu \times \nu|(A \cap(L \times Y)) \\
= & |\mu \times \nu|\left(A \cap \bigcup_{k=0}^{n}\left(S_{k} \times Y\right)\right) \\
= & \sum_{k=0}^{n}|\mu \times \nu|\left(A \cap\left(S_{k} \times Y\right)\right) \\
& \leq \sum_{k=0}^{n}|\mu \times \nu|\left(S_{k} \times V_{k}\right) \\
& \leq \sum_{k=0}^{n}|\mu|\left(S_{k}\right)|\nu|\left(V_{k}\right),
\end{aligned}
$$

pero

$$
\sum_{k=0}^{n}|\mu|\left(S_{k}\right)|\nu|\left(V_{k}\right) \leq \sum_{k=0}^{n}|\mu|\left(S_{k}\right) \epsilon=|\mu|\left(\bigcup_{k=0}^{n} S_{k}\right) \epsilon \leq|\mu|(S) \epsilon \leq \delta,
$$

es decir, $|\mu \times \nu|(A)<\delta$.

\section{Referencias}

[1] Berberian, S.K. (1967) Measure and Integration, Van Nostrand, Nueva York, 1965.

[2] Dinculeanu, N. (1967) Vector Measures. Pergamon Press, Nueva York. 
[3] Douwen, E.K. van (1989) "Fubini's theorem for null sets", Amer. Math. Monthly 96: 718-721.

[4] Fubini, G. (1907) "Sugli integrali multipli”, Rend. Accad. Lincei, Roma 16(1): 608-614. Reprinted in: Fubini, G. (1958) Opere Scelte, Vol. 2. Edizioni Cremonese, Unione Matematica Italiana/CNR, Roma. pp. 243-249.

[5] Halmos, P.R. (1974) Measure Theory. Springer-Verlag, Nueva York,

[6] Johnson, R.A. (1966) "On product measures and Fubini's theorem in locally compact spaces”, Trans. Amer. Math. Soc. 123: 112-129.

[7] Lebesgue, H.L. (1904) Leçons sur l'Intégration et la Recherche des Fonctions Primitives. Gauthier-Villars, Paris.

[8] Munkres, J.R. (2000) Topology. Prentice Hall, Upper Saddle River NJ.

[9] Oxtoby, J.C. (1971) Measure and Category. Springer-Verlag, Nueva York.

[10] Royden, H.L. (1988) Real Analysis, 3a edición. Macmillan Publishing Company, Nueva York.

[11] Rudin, W. (1979) Análisis Real y Complejo. Editorial Alhambra, Madrid. 